\title{
Conhecimento de trabalhadores de uma indústria acerca da prevenção do câncer de próstata
}

\author{
Knowledge of workers in an industry about the prevention of \\ prostate cancer
}

\author{
Francisco das Chagas Araújo Sousa' $\bullet$ Andreia Oliveira Elias Lima ${ }^{2} \bullet$ Wenderson Costa da Silva $^{3}$ \\ Pedro Gabriel Sobral da Silva ${ }^{4} \bullet$ Rogério Cruz Mendes $^{5} \bullet$ Eduardo Brito da Silva $^{6} \bullet$ Maria Gabrielle Sobral da Silva ${ }^{7}$ \\ Cristina Soares Oliveira ${ }^{8} \bullet$ Chrisllayne Oliveira da Silva ${ }^{9}$ Elide Andressa de Andrade Rodrigues Severo ${ }^{10}$ \\ Joyciara Vieira Almeida Moura" $\bullet$ Alicia Costa Santana ${ }^{12}$
}

\begin{abstract}
RESUMO
Objetivo: avaliar o conhecimento dos trabalhadores de uma fábrica de cera de carnaúba acerca da prevenção do câncer de próstata. Método:Trata-se de uma pesquisa de campo, descritiva exploratória, com uma abordagem quantitativa, a produção dos dados deu-se por meio da aplicação de questionário contendo perguntas de múltipla escolha. A coleta de dados resultou em um total de 25 homens entrevistados. Resultados: Quanto ao perfil dos participantes 12 (32\%) possuíam idade entre $3 \mathrm{I}$ a 40 anos e $4 \mathrm{I}$ a 50 anos respectivamente; sendo que 19 deles (76\%) possuíam cor parda; e a maioria eram casados (68\%). Em relação ao conhecimento e atitude acerca do câncer de próstata, $96 \%$ afirmam já ter ouvido falar da doença e conhecem alguma forma de detecção, afirmaram conhecer o toque retal, e $68 \%$ afirmaram nunca ter realizado nenhum exame de prevenção. Conclusão: Ressalta-se como fatores impactantes do câncer de próstata o exame do toque retal, a falta de interesse dos homens na prevenção dessa neoplasia e as condições emocionais do paciente frente ao diagnóstico desta patologia.
\end{abstract}

Palavras-chave: Enfermagem; Saúde do homem; Neoplasia Prostática Intraepitelial; Prevenção e Controle.

\begin{abstract}
Objective: to evaluate the knowledge of workers at a carnauba wax factory about the prevention of prostate cancer. Method:This is a descriptive, exploratory field research, with a quantitative approach, the data was produced through the application of a questionnaire containing multiple choice questions. Data collection resulted in a total of 25 men interviewed. Results: Regarding the profile of the participants, 12 (32\%) were aged between 31 to 40 years and 4 I to 50 years respectively; 19 of them $(76 \%)$ had brown skin; and the majority were married (68\%). Regarding knowledge and attitude about prostate cancer, $96 \%$ say they have heard of the disease and know some form of detection, said they know about digital rectal examination, and $68 \%$ said they had never had a preventive exam. Conclusion: Prostate cancer examination, the lack of interest of men in the prevention of this neoplasia and the emotional conditions of the patient when diagnosed with this pathology are highlighted as impacting factors of prostate cancer.

Key words: Nursing; Men's health; Intraepithelial Prostatic Neoplasm; Prevention and Control.
\end{abstract}

I Médico Veterinário, Doutor em Ciência Animal pela Universidade Federal do Piauí - UFPI. Professor Adjunto da Universidade Estadual do Piauí - UESPI E-mail: franciscoaraujo@ccs.uespi.br Teresina - PI

2 Graduada em Enfermagem pelo Centro Universitário do Piauí - UNIFAPI E-mail: andreiaoliveirajonatha@gmail.com Teresina - PI

3 Graduado em Enfermagem pelo Centro Universitário de Ciências e Tecnologia do Maranhão - UniFacema E-mail:wendersoncosta09@hotmail.com Caxias - MA

4 Graduando em Tecnólogo em radiologia do Centro Universitário de Ciências e Tecnologia do Maranhão - UniFacema E-mail: biielpedro707@gmail.com Caxias - MA

5 Graduado em Enfermagem pelo Centro Universitário de Ciências e Tecnologia do Maranhão - UniFacema E-mail: rogeriocruz82@yahoo.com.br Caxias - MA

6 Graduando em Enfermagem do Centro Universitário de Ciências e Tecnologia do Maranhão - UniFacema E-mail: eduzinhobds@gmail.com Caxias - MA

7 Graduanda em Enfermagem do Centro Universitário de Ciências e Tecnologia do Maranhão - UniFacema E-mail: mariagabrielle980@gmail.com Caxias - MA

8 Graduanda de Psicologia do Centro Universitário de Ciências e Tecnologia do Maranhão - UniFacema E-mail: wendersoncosta09@hotmail.com Caxias - MA

9 Bacharel em Enfermagem pelo Centro Universitário de Ciências e Tecnologia do Maranhão - UniFacema E-mail: wendersoncosta09@hotmail.com Caxias - MA

10 Bacharel em Enfermagem pelo Centro Universitário de Ciências e Tecnologia do Maranhão - UniFacema E-mail:wendersoncosta09@hotmail.com Caxias - MA

I I Bacharel em enfermagem pelo Centro Universitário de Ciência e Tecnologia do Maranhão - UniFacema E-mail: wendersoncosta09@hotmail.com Caxias - MA

12 Graduanda em enfermagem pelo Centro Universitário de Ciência e Tecnologia do Maranhão - UniFacema E-mail:wendersoncosta09@hotmail.com Caxias - MA 


\section{INTRODUÇÃO}

Câncer de próstata ou cancro da próstata é caracterizado pelo aumento exagerado da próstata, glândula que faz parte do sistema reprodutor masculino, localizada na parte baixa do abdômen ${ }^{(1)}$. O câncer de próstata é o segundo em incidência no mundo e a quinta causa de mortalidade por tumores malignos entre os homens ${ }^{(2)}$.

Em 20I2, ocorreram I4,I novos casos de câncer, 8,2 milhões de morte e 32,6 milhões de pessoas vivendo com esse agravo, em todo o mundo ${ }^{(3)}$. No Brasil, um país em desenvolvimento, o câncer de próstata é a segunda causa de mortalidade entre os homens, o Instituto $\mathrm{Na}$ cional do Câncer estimou que aproximadamente 69.000 novos casos dessa doença para os anos de 2014 e $2015^{(4)}$.

De 1996 a 2014, foram registrados na região nordeste do Brasil cerca de 46.089 óbitos por câncer de próstata, entre a faixa etária dos 50 a 80 anos, dos quais ocorreram 1.954 (4\%) óbitos na faixa etária de 50-59 anos, 7.616 óbitos (16\%) de 60-69 anos, 15.977 (35\%) óbitos dos 70-79 anos, e 20.542 (45\%) óbitos na faixa etária de 80 anos mais ${ }^{(5)}$.

O cancro da próstata $(\mathrm{CaP})$ aparece quando as células passam a se multiplicar de forma desorganizada, constituindo-se um tumor que na maioria das vezes pode ser maligno( ${ }^{(6)}$. O CaP é uma patologia de evolução lenta, com incidência elevada em homens com idade acima de 50 anos. Os principais sintomas mais comuns relacionados ao câncer de próstata, incluem hematúria, aumento do debito urinário à noite, jato urinário fraco, dor ou queimação ao urinar (2).

O diagnóstico do CaP é realizado por meio do exame digital da próstata e da dosagem do antígeno específico prostático (PSA). A detecção precoce é considera um importante fator de proteção para reduzir as taxas de morbidade e mortalidade.A precisão do resultado é meIhor quando realizado em conjunto com o toque retal(7-8).

As maiores barreiras quando se trata desta patologia é a falta de informação da população, com crenças antigas e negativas a respeito da $\mathrm{CaP}$ e seu prognóstico, pois existe um preconceito cultural em relação ao exame preventivo do toque retal, que gera sentimentos associados ao constrangimento e ao medo( ${ }^{(9)}$.

A detecção precoce é fundamental no combate contra o câncer de próstata, visto que, na fase inicial a doença apresenta uma evolução silenciosa. Além do mais, o combate do tumor em fase inicial tem $80 \%$ de chance de cura consequentemente uma melhor qualidade de $\mathrm{vida}^{(7)}$.

Nesse contexto, o profissional enfermeiro como integrante da equipe multiprofissional e atuante na Estratégia Saúde Família (ESF), considerada porta de entrada para o sistema de saúde, apresenta papel de destaque, atuando mediante adoção de práticas assistenciais, preventivas e de promoção à saúde ${ }^{(10)}$.
Dessa forma, torna-se fundamental não só falar sobre a temática, mas também, propor formas de ampliar as informações a respeito do câncer de próstata e, principalmente mostrar a importância da prevenção e da necessidade de seguir as medidas preventivas acerca desta patologia.

A relevância desse trabalho é conscientizar os trabaIhadores de uma fábrica de cera de carnaúba da importância da prevenção, e mostrar quais são os fatores de risco para o CaP, e expor o contexto do câncer de próstata na dimensão dos fatores de risco, sintomas, diagnósticos, tratamento, prevenção e dos aspectos culturais.

Partido dessas ideias este estudo sustentou-se na seguinte questão de pesquisa: Como se dá o conhecimento de trabalhadores de uma fábrica de cera de carnaúba acerca da prevenção do câncer de próstata?

Para tal, o objetivo desse estudo foi avaliar o conhecimento dos trabalhadores de uma fábrica de cera de carnaúba acerca da prevenção do câncer de próstata, bem como, a prática de exames na prevenção do câncer de próstata.

\section{MÉTODO}

Trata-se de uma de campo, descritiva, com abordagem quantitativa dos dados. $O$ estudo foi realizado em uma indústria de cera de carnaúba, localizada na BR II5, na cidade de Campo Maior (PI). O local da pesquisa foi escolhido por ser formado basicamente pela população masculina, alvo de nossa pesquisa.

A amostragem foi do tipo não probabilística por conveniência. A escolha dos referidos participantes teve como base a disponibilidade dos mesmos nas dependências da indústria nos momentos de coleta de dados.

Os critérios de inclusão da pesquisa foram: funcionários do sexo masculino, que possuir idade igual ou superior a 18 anos que assinou o Termo de Consentimento Livre e Esclarecido (TCLE). Os critérios de exclusão foram: funcionários que estavam de férias ou licença, afastado das atividades laborais.

A coleta de dados ocorreu nos meses de setembro e outubro de 2019. Como instrumento para coleta de dados da pesquisa utilizou-se um formulário que abrangeu variáveis sociodemográficas (idade, escolaridade, situação conjugal, renda e religião), além dos hábitos de vida dos participantes. E um questionário composto de perguntas abertas e fechadas, as quais tiveram como base outras de questionários de pesquisas nacionais e internacionais abrangeu variáveis como: conhecimentos, atitudes e prática relacionada ao exame da próstata.

A análise dos resultados obtidos foi processada por intermédio de cálculos estatísticos, colocados em números absolutos e percentuais. Os dados foram armazenados em planilha do programa Microsoft Office 
Excel $\AA$ for Windows $\AA$ e dispostos em tabelas de análise descritiva simples.

O Projeto de pesquisa foi submetido à Plataforma Brasil e direcionado ao Comitê de Ética em Pesquisa da Universidade Paulista (CEP/UNIP). E aprovado com $N^{\circ}$ de CAAE I7527|19.2.000055।2. O desenvolvimento do estudo seguiu todos os procedimentos éticos de pesquisa, pleiteando as técnicas adequadas descritas na literatura para o cumprimento dos preceitos éticos relacionados à pesquisa com seres humanos, estabelecidos pela resolução 466/12 do Conselho Nacional de Saúde (CNS). Assim sendo, a coleta de dados desse estudo somente foi realizada após ter sido aprovada pelo CEP.

\section{RESULTADOS}

A coleta de dados resultou em um total de 25 homens entrevistados nesse estudo. Quanto ao perfil dos participantes, I 2 (32\%) possuíam idade entre 31 a 40 anos e $4 \mathrm{I}$ a 50 anos respectivamente; sendo que $19(76 \%)$ deles possuíam cor parda e a maioria eram casados (68\%). Verificou-se que 20 (80\%) dos indivíduos entrevistados possuem ensino fundamental incompleto, 25 (100\%) não possuíam plano de saúde, e 22 (88\%) declararam ter fiIhos e ser católicos. Em relação ao estilo e hábitos de vida dos participantes, 22 (88\%) afirmaram não ser fumantes, em contrapartida 19 (76\%) informaram ser etilistas e $13(52 \%)$ declararam não praticarem exercícios físicos (Tabela 0I).

Em relação ao conhecimento acerca do câncer de próstata, 24 (96\%) afirmaram já ter ouvido falar da doença. Quanto ao meio que ouviram falar, entre 25 (100\%) entrevistados, 17 (68\%) identificaram a imprensa como o principal veículo desta informação; seguido de $05(20 \%)$ referente a outros serviços de saúde. Quando indagados sobre se conhecem alguma forma de detecção do câncer, 15 (60\%) referiram conhecer. Desses, 10 (40\%) conheciam o toque retal. Além disso, $19(76 \%)$ referiram a idade adequada para o rastreamento do câncer de próstata como sendo de 40 a 50 anos, conforme descrito na Tabela 02.

A Tabela 03 apresenta as opiniões dos participantes acerca da detecção do câncer de próstata, entre as variáveis analisadas, constatou-se que $16(64 \%)$ dos entrevistados consideraram o exame de próstata como a

TABELA 1 - Caracterização sociodemográfica dos participantes do estudo (n=25). Campo Maior, PI, Brasil, 2019

\begin{tabular}{|c|c|c|}
\hline Variáveis sociodemográficas & $\mathbf{N}$ & $\%$ \\
\hline \multicolumn{3}{|l|}{ Faixa etária } \\
\hline $18-30$ anos & 05 & 20,0 \\
\hline $31-40$ anos & 08 & 32,0 \\
\hline $41-50$ anos & 08 & 32,0 \\
\hline$>50$ anos & 04 & 16,0 \\
\hline \multicolumn{3}{|l|}{ Cor } \\
\hline Branca & 01 & 4,0 \\
\hline Negra & 05 & 20,0 \\
\hline Parda & 19 & 76,0 \\
\hline \multicolumn{3}{|l|}{ Situação Conjugal } \\
\hline Casado & 17 & 68,0 \\
\hline Solteiro & 08 & 32,0 \\
\hline Divorciado & - & - \\
\hline Viúvo & - & - \\
\hline \multicolumn{3}{|l|}{ Escolaridade } \\
\hline Fundamental incompleto & 20 & 80,0 \\
\hline Fundamental completo ou mais & 05 & 20,0 \\
\hline Nunca estudou & - & - \\
\hline \multicolumn{3}{|l|}{ Possui Plano de Saúde } \\
\hline Sim & - & - \\
\hline Não & 25 & 100,0 \\
\hline \multicolumn{3}{|l|}{ Possui Filhos } \\
\hline Sim & 20 & 80,0 \\
\hline Não & 05 & 20,0 \\
\hline \multicolumn{3}{|l|}{ Religião } \\
\hline Católicos & 22 & 88,0 \\
\hline Evangélicos & 02 & 8,0 \\
\hline Espirita & 01 & 4,0 \\
\hline Não possui religião & - & - \\
\hline \multicolumn{3}{|l|}{ Tabagista } \\
\hline Sim & 03 & 12,0 \\
\hline Não & 22 & 88,0 \\
\hline \multicolumn{3}{|l|}{ Etilista } \\
\hline Sim & 19 & 76,0 \\
\hline Não & 06 & 24,0 \\
\hline \multicolumn{3}{|l|}{ Pratica Exercícios Físicos } \\
\hline Sim & 12 & 48,0 \\
\hline Não & 13 & 52,0 \\
\hline Total & 25 & 100,0 \\
\hline
\end{tabular}

Legenda: N=número; \%=percentual.

Fonte: Pesquisa direta, 2019. 
TABELA 02 - Conhecimento acerca do câncer de próstata avaliados em homens de uma fábrica de cera de carnaúba (n=25). Campo Maior, PI, Brasil, 2019.

\begin{tabular}{|c|c|c|}
\hline Variáveis & $\mathbf{N}$ & $\%$ \\
\hline \multicolumn{3}{|c|}{ Já ouviu falar sobre o câncer de próstata } \\
\hline Sim & 24 & 96,0 \\
\hline Não & 01 & 4,0 \\
\hline \multicolumn{3}{|l|}{ Por qual meio ouviu falar } \\
\hline TV/rádio/Jornal & 17 & 68,0 \\
\hline Amigos & - & - \\
\hline ESF & 01 & 4,0 \\
\hline Outros serviços de saúde & 05 & 20,0 \\
\hline Parentes & 02 & 8,0 \\
\hline \multicolumn{3}{|l|}{ Outros } \\
\hline \multicolumn{3}{|c|}{ Conhece algum exame de detecção do câncer } \\
\hline Sim & 15 & 60,0 \\
\hline Não & 10 & 40,0 \\
\hline \multicolumn{3}{|l|}{ Quais exames conhecem } \\
\hline Toque retal & 10 & 40,0 \\
\hline Exame de sangue & 05 & 20,0 \\
\hline Outros & - & - \\
\hline \multicolumn{3}{|c|}{ Na sua opinião em que idade os homens deveriam se preocupar mais em fazer os exames } \\
\hline$<40$ anos & 04 & 16,0 \\
\hline $40-50$ anos & 19 & 76,0 \\
\hline$>50$ & 02 & 8,0 \\
\hline Não Sabem & - & - \\
\hline Total & 25 & 100,0 \\
\hline
\end{tabular}

Legenda: N=número; \%=percentual.

Fonte: Pesquisa direta, 2019.

TABELA 03 - Opiniões (atitudes) em relação a detecção do câncer de próstata entre os entrevistados de uma fábrica de cera de carnaúba (n=25). Campo Maior, PI, Brasil, 2019

\begin{tabular}{|c|c|c|}
\hline Variáveis & $\mathbf{N}$ & $\%$ \\
\hline \multicolumn{3}{|c|}{ A única forma de suspeitar do câncer é através do exame de próstata } \\
\hline Sim & 16 & 64,0 \\
\hline Não & 09 & 36,0 \\
\hline \multicolumn{3}{|c|}{ Qual a frequência adequada de realização do exame } \\
\hline Anualmente & 16 & 64,0 \\
\hline A cada 2 anos & 07 & 28,0 \\
\hline A cada 3 a 5 anos & - & - \\
\hline A cada 5 anos & - & - \\
\hline Somente quando tiverem os sintomas & - & - \\
\hline Não deveriam fazer & - & - \\
\hline Não soube responder & 02 & 8,0 \\
\hline \multicolumn{3}{|c|}{ Só deveria fazer o exame os homens que tiveram sintomas urinários } \\
\hline Sim & 02 & 8,0 \\
\hline Não & 22 & 88,0 \\
\hline Não soube responder & 01 & 4,0 \\
\hline \multicolumn{3}{|c|}{ Importância de fazer o exame de próstata regularmente } \\
\hline Muito Importante & 20 & 80,0 \\
\hline Importante & 05 & 20,0 \\
\hline Indiferente & - & - \\
\hline Pouco ou nada importante & - & - \\
\hline Total & 25 & 100,0 \\
\hline
\end{tabular}

Legenda: N=número; \%=percentual.

Fonte: Pesquisa direta, 2019.

única forma de suspeição diagnóstica. Quanto a frequência adequada para a realização desse exame, 16 (64\%) afirmaram que deve ser anualmente; e quando indagados se só deveria fazer o exame os homens com sintomas urinários, 22 (88\%) afirmaram que não. Já quanto a im- portância dada ao exame de próstata, 20 (80\%) referiram como "muito importante" e 05 (20\%) como "importante", respectivamente.

No que diz respeito às práticas de rastreamento do câncer de próstata descritos na Tabela 04, 14 (56\%) dos 
TABELA 04 - Práticas em relação aos exames de rastreamento do câncer de próstata entre os entrevistados na fábrica de cera de carnaúba em Campo Maior, PI, Brasil, 2019. (N=25)

\begin{tabular}{|c|c|c|}
\hline Variáveis & $\mathbf{N}$ & $\%$ \\
\hline \multicolumn{3}{|c|}{ Algum medico já informou que deveria fazer exame de próstata } \\
\hline Sim & 11 & 44,0 \\
\hline Não & 14 & 56,0 \\
\hline Não lembram & - & - \\
\hline \multicolumn{3}{|l|}{ Já realizou exame de próstata } \\
\hline Sim & 08 & 32,0 \\
\hline Não & 17 & 68,0 \\
\hline Não lembram & - & - \\
\hline \multicolumn{3}{|l|}{ Se sim quais os motivos } \\
\hline Tinham sintomas & - & - \\
\hline Caso de câncer na família & 01 & 4,0 \\
\hline Rotina de prevenção & 07 & 28 \\
\hline Outro motivo & - & - \\
\hline \multicolumn{3}{|c|}{ Se sim qual foi a última vez que realizou } \\
\hline Há menos de 1 ano & 03 & 12,0 \\
\hline Entre 1 e 2 anos & 01 & 4,0 \\
\hline Entre 3 e 5 anos & 03 & 12,0 \\
\hline Há mais de 5 anos & 01 & 4,0 \\
\hline Total & 25 & 100,0 \\
\hline
\end{tabular}

Legenda: N=número; \%=percentual.

Fonte: Pesquisa direta, 2019.

homens referiram que nenhum médico lhes havia informado que deveriam fazer o exame de próstata; 17 (68\%) nunca realizaram o exame, e dos 08 (32\%) dos indivíduos que já realizaram, o principal motivo de solicitação pelo profissional foi apontado por 07 (28\%) como sendo "rotina de prevenção”. Ressalta-se que 03 (12\%) realizaram o exame há menos de um ano, e entre três e cinco anos.

\section{DISCUSSÃO}

Este estudo avaliou as condições sociodemográficas de trabalhadores de uma fábrica de cera de carnaúba, bem como conhecimento sobre doença oncológica, atitudes em relação a detecção do câncer e práticas em relação aos exames de rastreamento.

Considerando as características sociodemográficas, a faixa etária de 30 a 50 anos pode ser considerada de risco para o desenvolvimento da doença, ainda mais se tiverem um fator agravante como nunca ter realizado exames preventivos e de rastreamento ${ }^{(2)}$.Apesar do câncer de próstata ser mais prevalente na terceira idade, sendo que $62 \%$ dos casos de câncer de próstata no mundo surgem com 65 anos ou mais ${ }^{(3)}$. Em alguns casos o indivíduo pode ser assintomático, por isso a importância de realizar os exames de rastreamento, bem como o acompanhamento com um profissional da saúde ${ }^{(7)}$.

Quanto a etnia não foram encontrados dados concisos na literatura que estabelecessem uma relação entre etnia e a patologia, mas acredita-se que os riscos para o desenvolvimento de câncer podem variar de diferentes raças ou etnias, essas diferenças podem estar relacionadas a características genéticas, estilo de vida e ambien- te $\mathrm{e}^{(\mathrm{II})}$. Porém tende a se apresentar em maior número em homens com etnia branca seguidos de pardos ${ }^{(3)}$. Em relação a situação conjugal acredita-se que este seja um fator positivo, pois favorece que os homens procurarem atendimento médico e realizem os exames de rastreamento, pois são incentivados pelos seus cônjuges (2).

O nível de educação dos participantes deste estudo corroboram com os dados encontrados nos estudos de Fernandes et al. ${ }^{(3)}$ em que $57,4 \%$ dos entrevistados não possuíam ensino fundamental completo e em Ribeiro et al. ${ }^{(2)}$ obteve em suas amostras que $46 \%$ do entrevistados não tinha o primeiro ano do ensino médio completo. Esses dados revelam que o nível de escolaridade influência no nível de conhecimento e percepção dos indivíduos acerca do câncer de próstata, pois estes indivíduos tendem a levar em consideração as informações advindas do meio familiar e cultural (12).

Sobre à adoção de hábitos prejudiciais à saúde, observou-se que o uso da bebida alcoólica é mais frequente que o uso de tabaco, conforme relata Rodrigues ${ }^{(13)}$ em seu estudo o consumo de bebidas alcoólicas em excesso, tabagismo, além da alta ingestão de gorduras e carnes vermelhas estão associados ao desenvolvimento de várias doenças crônicas degenerativas, incluindo o câncer de próstata, tendo em vista que estes hábitos possuem um potencial mutagênico considerável para o surgimento dessas patologias.

No que se refere ao peso corporal, a maioria dos entrevistados afirmaram não praticar exercícios físicos, entretanto o Instituto Nacional do Câncer ${ }^{(14)}$ aponta que o excesso de peso corporal está associado ao maior risco de câncer de próstata avançado, desta forma falta de 
exercícios físicos influência diretamente para o desenvolvimento da doença. Mas uma rotina que inclua a prática de exercícios físicos associados a uma dieta equilibrada favorece a prevenção do câncer de próstata( ${ }^{(7)}$.

Relacionado ao nível de conhecimentos dos trabaIhadores sobre câncer de próstata, dados na literatura corroboram com os resultados encontrados neste estudo, em pesquisa realizada por Ribeiro et al.(2) todos os entrevistados afirmaram ter certo conhecimento sobre câncer de próstata e prevenção por meio de TV/ rádio, familiares, amigos e palestras, no entanto não sabiam explicar os métodos de prevenção e os exames de detecção precoce. E em concordância Fernandes et al.(3) ressaltam que tendo um conhecimento prévio em relação à doença como antecedentes familiares,-sintomas e fatores predisponentes, poderia influenciar a prática do autocuidado nos homens.

Porém, ainda existe uma carência de informações entre os homens sobre o câncer de próstata e como preveni-lo, em sua maioria afirmam que sabem um pouco sobre a doença, mas não sabem sobre os métodos de prevenção, o que pode tornar esses indivíduos favoráveis para que a doença se instale, muitas vezes de início assintomática, ou uma vez detectada em um estágio avançado, na qual na grande maioria dos casos resulta em óbito ${ }^{(7)}$.

Quanto ao exame de rastreamento para o câncer de próstata, dados desta pesquisa corroboram com os estudos de Figueredo ${ }^{(7)}$ em que destaca que os homens tem conhecimento em relação a idade para iniciar os exames preventivos, mas a maioria não realiza. Em uma estimativa do Instituto Nacional de Câncer (INCA), o câncer de próstata apresenta uma incidência de $29,2 \%$ de casos, é importante ressaltar que alguns desses tumores podem crescer de forma rápida e se espalhar para outros órgãos e podendo levar à óbito, e em outros casos podem crescer de forma tão lenta (+/- 15 anos para atingir I $\mathrm{cm}^{3}$ ) não apresentando sinais durante a vida que possam colocar em risco a saúde do homem ${ }^{(15)}$.

O exame de toque retal é uma medida preventiva de baixo custo, no entanto, o medo de ser tocado e de sentir dor, ou eventual ereção que possa ser confundida com prazer, são alguns fatores que impedem o homem a procurar os serviços de saúde, associados muitas vezes ao despreparo dos profissionais de saúde em abordar os aspectos simbólicos que permeiam o imaginário masculino para realização deste exame ${ }^{(9)}$. Porém o toque retal é visto como uma ameaça e violação ao ser masculino, pois a próstata é como algo simbólico para os homens, assim os fatores sociais e culturais que permeiam o ser masculino, como um ser forte e viril, podem se sobressair a sentimentos de medo e receio quando apresentam sintomas do câncer de próstata ${ }^{(12)}$.

Ressalta-se que não é somente o desconhecimento sobre a doença que pode acarretar implicações para o paciente, mas também o baixo nível de renda e principalmente o medo, que colaboram para a não detecção precoce o que resulta em um diagnóstico tardio, ocasionando uma taxa elevada de mortalidade ${ }^{(16)}$.

Tendo em visto como o câncer de próstata ainda é um grande problema de saúde pública, o profissional de enfermagem e equipe interdisciplinar devem direcionar ações educativas em saúde, pois o câncer de próstata envolve muito mais do que a fisiopatologia da doença, mas também aspectos emocionais, sociais e culturais ${ }^{(9)}$. Tais ações devem contribuir para a transformação e mudanças na prática assistencial preventiva, voltada para percepção dos homens e sua singularidade com ênfase nos cuidados de saúde, reduzindo o medo e o constrangimento dos homens ${ }^{(16)}$.

Dessa forma, acredita-se que os profissionais de saúde devem desenvolver atividades educativas, visando um maior esclarecimento sobre a doença e, principalmente, sobre as medidas preventivas, pois essa desinformação é preocupante, já que o conhecimento sobre a doença e os meios de preveni-la podem ser decisivos sobre sua evolução(7).

\section{CONCLUSÃO}

Com base nos resultados, observa-se a necessidade de atividades como de educação em saúde acerca do câncer de próstata, e assim contribuir de forma significativa para a redução do percentual de diagnósticos em estágios avançados da doença. Dessa forma, estratégias básicas se tornam imprescindíveis, como atividades dinâmicas em que são repassadas de forma simples e acessível respeitando a escolaridade e os padrões de valores do homem. É fundamental que essas atividades estejam pautadas na mudança de comportamento, tanto por parte dos homens, bem como, dos provedores de serviços de saúde, priorizando os exames de rastreamento do câncer de próstata.

Nesse contexto, é preciso levar em consideração os aspectos estruturais e simbólicos que perpassam o toque retal, que a falta de tempo e o preconceito estão intimamente relacionados à ausência de interesse por parte dos homens com relação à prevenção do câncer de próstata.

Em relação as limitações desse estudo, a primeira relaciona-se com amostra do estudo em questão, por ter sido restrito a uma única indústria, o que dificulta a generalização dos resultados. A segunda por se tratar de um estudo com delineamento transversal, no qual não foi possível definir uma relação de causalidade.

Espera-se que esses resultados contribuem para um maior conhecimento profissional, permitindo uma reflexão sobre a abordagem do câncer de próstata, contribuindo com informações pertinentes à sociedade, visando aumentar a adesão aos exames de detecção precoce do câncer de próstata. 


\section{REFERÊNCIAS}

I. Bacelar Júnior AJ, Menezes CS, Barbosa CA, Freitas GBS, Silva GG,Vaz JPS, et al. Câncer de próstata: métodos de diagnósticos. prevenção e tratamento. Brazilian Journal of Surgery and Clinical Research- BJSCR [Internet]. 2015 [acesso em 17 mar 2019]; 10(3):40-46. Disponível em: https://www. mastereditora.com.br/periodico/20I5050I_I74533.pdf.

2. Ribeiro LS, Lubenow JAM, Silva PE, Correia AA. Conhecimento de homens acerca da prevenção do câncer de próstata. Revista ciências saúde [Internet]. 2015 [acesso em 23 jun 20I9] 13(2):4-I0. Disponível em: http://www.facene. com.br/wp-content/uploads/2010/I I/Conhecimento-de -homens-PRONTO.pdf.

3. Fernandes MV, Martins JT, Cardelli AAM, Marcon SS, Ribeiro RP. Perfil epidemiológico do homem com câncer de próstata atendido em um hospital universitário. Cogitare enfermagem [Internet]. 2014 [acesso em 23 abr 2019]; 19(2):333-340. Disponível em: https://revistas.ufpr.br/cogitare/article/view/3 I540.

4. Bandeira TC, Deluca J, Beal R, Silva CM, Peder LD de. câncer de próstata: epidemiologia e associação com infecção urinária. Revista saúde.com [Internet]. 2018 [acesso em 30 abr 2019]; 14(3):।245-125।. Disponível em: http://periodicos2.uesb.br/index.php/rsc/article/view/42I7.

5. Sousa BOA, Rossato R, Júnior PMR, Bitencourt EL. Incidência de câncer de próstata no estado do Tocantins entre 200 I e 20I5. Revista de Patologia do Tocantins [Internet]. 2018 [acesso em 14 fev 2019] 5(2): 14-20. Disponível em: https://sistemas.uft.edu.br/periodicos/index.php/patologia/ article/download/5 I45//3998/.

6. Oliveira MM de, Malta DC, Guauche H, Moura L de, Silva GA. Estimativa de pessoas com diagnóstico de câncer no Brasil: dados da Pesquisa Nacional de Saúde, 2013. Rev. bras. epidemiol. [Internet]. 2015 [acesso em 24 fev 2019]; I8(Suppl 2):|46-I57. Disponível em: http://www.scielo.br/scielo.php?script=sci_arttext\&pi$\mathrm{d}=\mathrm{S}|4| 5-790 \times 20|5000600| 46 \& \mathrm{lng}=\mathrm{en} . \quad \mathrm{https} / / \mathrm{doi}$. org/I0.1590/1980-549720I5000600/3.

7. Figueredo AFS, Durães LE, Junior RFS, Barbosa HAB, Silva CSO. Percepção dos homens em relação a prevenção do câncer de próstata. Humanidades [Internet]. 2014 [acesso em 23 ago 2019]; 3(2): 60-69. Disponível em: http://www. revistahumanidades.com.br/arquivos_up/artigos/a l20.pdf.

8. Furine AAC, Silva FA da, Santos FCB dos, Sanches MH, Machado RLD. Nível sérico de antígeno prostático específico em usuários de um laboratório clínico de Novo Horizonte, São Paulo. Revista Brasileira de analises clinicas [Internet]. 2016 [acesso em 23 mar 2019]. Disponível em: http://www. rbac.org.br/artigos/nivel-serico-de-antigeno-prostatico-es- pecifico-em-usuarios-de-um-laboratorio-clinico-de-novo -horizonte-sao-paulo/.

9. Belinelo RGS, Almeida SM de, Oliveira PP de, Onofre PSC, Viegas SMF, Rodrigues AB. Exames de rastreamento para o câncer de próstata: vivência de homens. Esc.Anna Nery [Internet]. 2014 Dec [acesso em 23 mar 2019]; 18(4):697-704. Disponível em: http://www.scielo.br/scielo.php?script=sci_ arttext\&pid=S|4|4-8|4520 | 4000400697\&Ing=en. https:// doi.org/| 0.5935/|4|4-8|45.20|40099.

10. Albuquerque GA, Leite MF,Belém JM, Nunes JFC, Oliveira MA de,Adami F. O homem na atenção básica: percepções de enfermeiros sobre as implicações do gênero na saúde. Esc.Anna Nery [Internet]. 2014 [acesso em 23 nov 20I9]; I8(4):607614. Disponível em: http://www.scielo.br/scielo.php?script=sci_arttext\&pid=SI4|4-8|4520|4000400607\&Ing=en. https://doi.org/|0.5935/|4|4-8|45.20|40086.

II. Oliveira PMP, Lima MMF, Cezario KG, Oliveira MG. Conhecimento de trabalhadores sobre o câncer de próstata: estudo descritivo exploratório. Online Brazilian Journal of Nursing [Internet]. 2012 [acesso em 25 ago 2020]; II(I). Disponível em: http://www.objnursing.uff.br/index.php/nursing/article/view/3595/html_I.

12. Amthauer C. As representações da masculinidade na adesão do toque retal como prevenção contra o câncer prostático. Rev Fund Care Online [Internet]. 2016 [acesso em 25 agosto]; 8(3): 4733-4737. Disponível em: http:// www.seer.unirio.br/index.php/cuidadofundamental/article/ view/3584.

13. Rodrigues RS, Sá RG, Zangrande C. Perfil epidemiológico dos portadores de câncer de próstata em tratamento na unidade de referência em oncologia do município de Sinop/ MT. Enfermagem Brasil [Internet]. 2016 [acesso em 25 ago 2020]; I5(4). Disponível em: https://portalatlanticaeditora. com.br/index.php/enfermagembrasil/article/view/497// 004.

14. Brasil. Ministério da Saúde. Instituto nacional do câncer. Incidência do câncer no Brasil: Estimativa 2014. Rio de Janeiro: INCA; 2014.

15. Brasil. Instituto Nacional de Câncer. ABC do câncer: abordagens básicas para o controle do câncer. Rio de Janeiro: INCA; 2020.

16. Krüger FPG, Cavalcanti G. Conhecimento e Atitudes sobre - Câncer de Próstata no Brasil: Revisão Integrativa. Revista Brasileira de Cancerologia [Internet]. 2018 [acesso em 25 ago 2020]; 64(4): 56I-567. Disponível em: http://docs.bvsalud.org/biblioref/2019/1 I/1025/59/conhecimento-e-atitudes-sobre-o-cancer-de-prostata-no-brasil-r_CB8sZdb.pdf.

Recebido: 2020-02-28

Aceito: 2020-09-04 